УДК 66.067 .55

\title{
ИНТЕЛЛЕКТУАЛЬНАЯ ИНФОРМАЦИОННО-ИЗМЕРИТЕЛЬНАЯ СИСТЕМА ТЕПЛОФИЗИЧЕСКИХ СВОЙСТВ ТЕПЛОИЗОЛЯЦИОННЫХ ПОКРЫТИЙ МАГИСТРАЛЬНЫХ ТРУБОПРОВОДОВ
}

\author{
Селиванова Зоя Михайловна', \\ selivanova@mail.jesby.tstu.ru \\ Павлов Владимир Иванович', \\ vpavl@mail.ru \\ 1 Тамбовский государственный технический университет,
Россия, 392000, г. Тамбов, ул. Советская, 106.
}

Актуальность. В магистральных трубопроводах для транспортировки, а также в резервуарах для хранения нефти и газа применяются теплозащитные покрытия из различных материалов. Теплозащита данных объектов неизбежно интенсивно подвергается воздействию различных климатических факторов, а в некоторых случаях механическим воздействиям, что приводит к деградации ее свойств. Разработка интеллектуальной информационно-измерительной системы с реконфигурируемой структурой для оперативного контроля состояния теплоизоляционных материалов, позволяющей расширить функциональные возможности существующих аналогичных систем и обеспечить требуемую точность измерений, является важной и актуальной.

Цель: повысить точность определения коэффициента теплопроводности различных теплозащитных покрытий магистральных трубопроводов и резервуаров при их производстве и эксплуатации для предотвращения ухудшения свойств теплоизоляции под воздействием внешних влияющих факторов.

объекты: теплозащитные покрытия магистральных трубопроводов и резервуаров, используемых при транспортировке и хранении нефти и газа.

Методы. Методологическую основу при решении поставленных задач проектирования интеллектуальной информационно-измерительной системы теплофизических свойств материалов составляют: теория измерительных систем, методы искусственного интеллекта, байесовский подход.

Результаты. Разработана концептуальная модель проектирования интеллектуальной информационно-измерительной системы теплофизических свойств теплоизоляционных покрытий магистральных трубопроводов для транспортировки нефти и газа и резервуаров при хранении. В рамках концептуальной модели разработана методика определения измерительной ситуации в процессе теплофизических измерений на основе предварительной идентификации свойств исследуемого материала с использованием байесовского подхода к обработке выходных сигналов первичных измерительных преобразователей и структурных компонентов измерительного канала системы. Разработана модель процесса реконфигурирования и соответствующая структура интеллектуальной информационно-измерительной системы теплофизических свойств теплоизоляционных покрытий с целью повышения точности определения коэффициента теплопроводности теплоизоляционных материалов. Относительная погрешность измерения коэффициента теплопроводности с применением предложенной системы составляет не более 5 \%.

\section{Ключевые слова:}

Концептуальная модель проектирования, байесовский подход,

модель процесса измерения, магистральный трубопровод, резервуар.

\section{Введение}

Применение теплоизоляции трубопроводов необходимо в зимний период для перекачки высокопарафинистой и вязкой нефти, исключения конденсата, сокращения теплопотерь, уменьшения осадки трубопроводов, которые прокладываются в пучинистых грунтах при транспортировке охлажденных газов, для защиты окружающей среды от теплового воздействия трубопроводов. Для теплоизоляции трубопроводов используются следующие материалы: минеральные ваты, маты из стеклянного волокна, пенопласты, рубероиды, пенобетоны, пенополиуретаны и др. с теплопроводностью от 0,02 до 0,2 Вт/мК [1]. При выборе тепловой изоляции, а также в процессе эксплуатации трубопроводов необходимо контролировать теплофизические свойства (теплопроводность, температуропроводность и теплоемкость) применяемых теплоизоляционных материалов, так как на теплозащитное покрытие оказывают негативное влияние внешние дестабилизирующие факторы.
В качестве измерительных средств указанных параметров теплофизических свойств (ТФС) широко применяются информационно-измерительные системы (ИИС). Однако, как показывает практика их применения, ИИС имеют ряд существенных недостатков: отсутствие требуемой точности измерений при воздействии влияющих факторов, а также возможности выбора метода контроля и адаптации в условиях неопределенности к измерительной ситуации [2]. Для повышения эффективности ИИС предлагается их интеллектуализация в результате использования методов искусственного интеллекта для проектирования интеллектуальных ИИС (ИИИС), которые позволяют выполнять интеллектуальные процедуры принятия решений по выбору математической модели ИМ, метода определения ТФС материалов в соответствии с моделью процесса измерения, оптимальных режимных параметров измерений, т. е. формированию измерительной ситуации в соответствии с классом исследуемых материалов (ИМ) по теплопроводности и 
допустимой относительной погрешностью измерений не более $5 \%$ [3-5].

Постановка задач проектирования ИИИС ТФС материалов. При проектировании эффективных ИИИС ТФС исследуемых материалов необходимо решить следующие задачи: разработать концептуальную модель проектирования ИИИС ТФС материалов с реконфигурируемой структурой; разработать модель процесса реконфигурирования структуры ИИИС ТФС материалов; решить задачу идентификации свойств исследуемого материала на основании байесовского подхода к обработке выходных сигналов первичных измерительных преобразователей (измерительных датчиков) и других структурных компонентов измерительного канала ИИИС (усилителя и аналого-цифрового преобразователя) для последующей идентификации и формирования измерительной ситуации; разработать модель процесса измерения в ИИИС ТФС материалов; создать структурную схему ИИИС ТФС материалов, реконфигурируемую в зависимости от результатов идентификации измерительной ситуации [6, 7].

Разработанная кониептуальная модель проектирования ИИИС ТФС материалов (рис. 1) включает основные компоненты, которые содержат информацию о предметной области, задачах и стратегиях создания ИИИС ТФС материалов, математических моделях, ограничениях, измерительных ситуациях, а также проблемах и рисках при проектировании системы. Созданная концептуальная модель отражает системные свойства [8]: преобразование структурных компонентов системы согласно из- мерительной ситуации; применение средств автоматизации, типизация технических характеристик и структуры при проектировании системы. В соответствии с концептуальной моделью основными рисками в процессе проектирования ИИИС ТФС материалов являются: определение параметров ТФС теплоизоляционных материалов и оценка относительной погрешности измерений выполняется только при создании опытного образца и проведении его экспериментальных исследований; при несоответствии погрешности результатов измерения ИИИС заданным в техническом задании на проектирование системы (не более 5 \%) необходимо дополнительное финансовое вложение на доработку ИИИС.

Концептуальная модель отличается возможностью использования её для различных предметных областей применения ИИИС; адаптации структуры системы к различным измерительным ситуациям, которые включают математические модели исследуемых материалов, методы контроля ТФС материалов, режимные параметры проведения теплофизических измерений. Концептуальная модель позволяет разработать информационную и функциональную модели ИИИС, базу знаний и данных, принимать решения для реконфигурирования структурных компонентов ИИИС.

Создана модель процесса реконфигурирования структуры ИИИС ТФС материалов. Системный подход и теория графов $[9,10]$ позволяют представить в виде графа математическую модель процесса реконфигурирования структуры ИИИС (рис. 2).

Реконфигурирование типовой структуры ИИИС ТФС материалов осуществляется в зависимо-

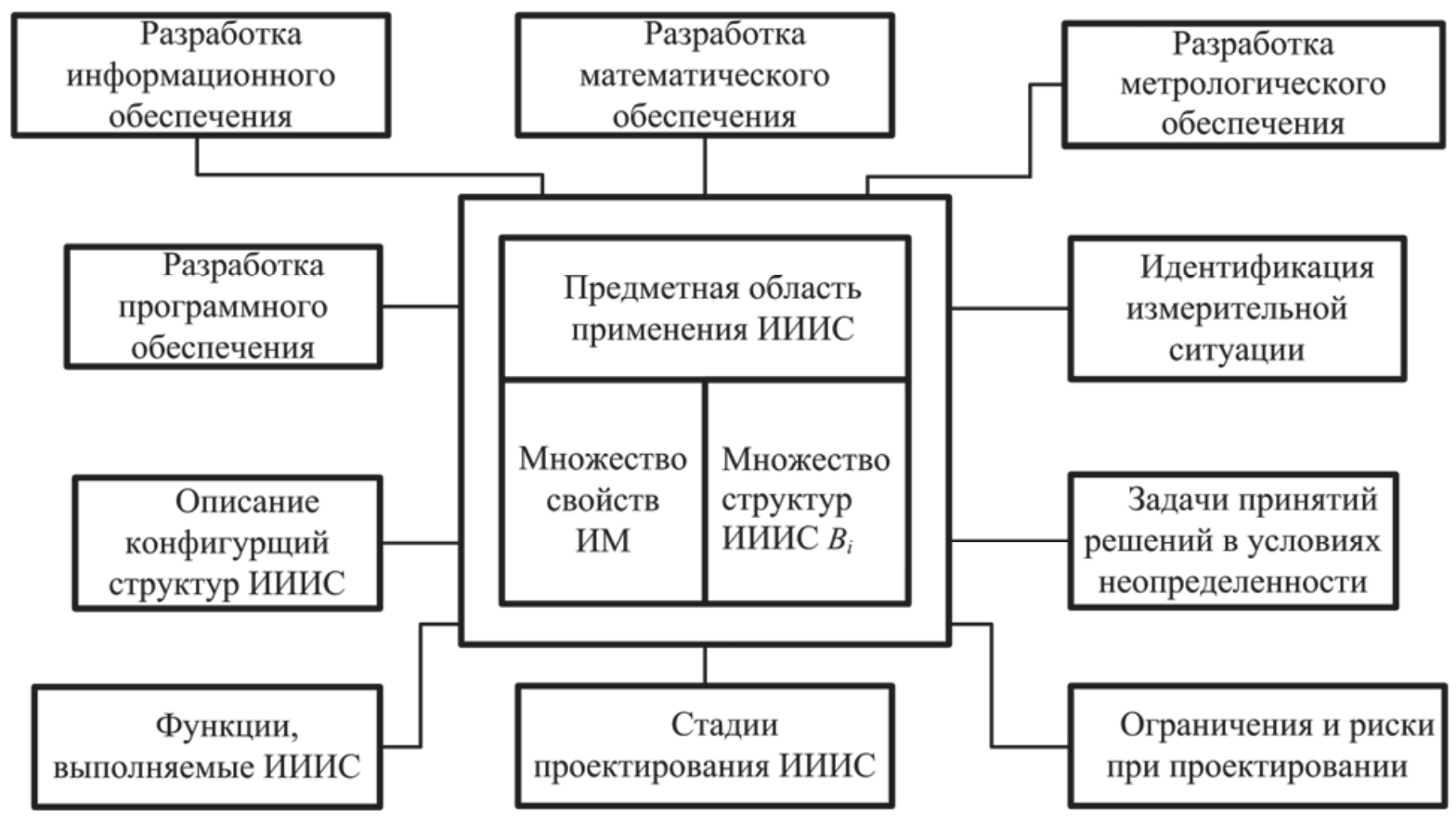

Рис. 1. Концептуальная модель проектирования ИИИС ТФС материалов с реконфигурируемой структурой

Fig. 1. Conceptual model of design of intellectual information-measuring system of thermophysical properties of materials with reconfigurable structure 


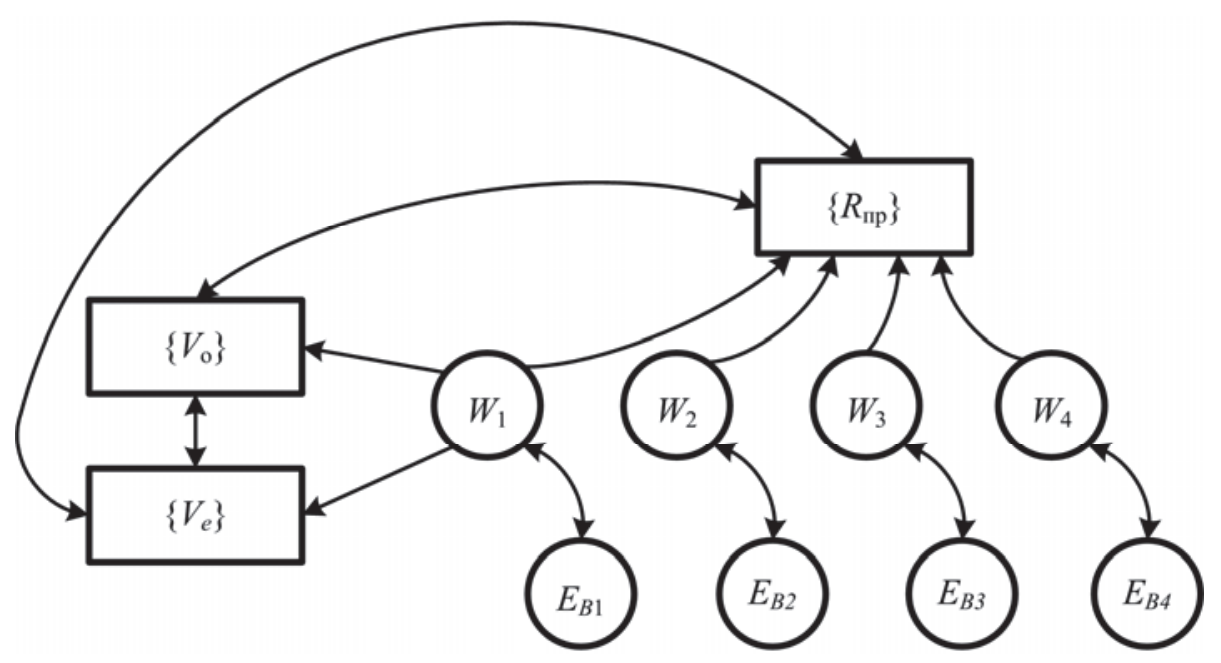

Рис. 2. Модель процесса реконфигурирования структуры ИИИС ТФС материалов

Fig. 2. Model of reconfiguring the structure of IIIS TFS of materials

сти от результатов идентификации измерительной ситуации с использованием модели процесса реконфигурирования структуры ИИИС ТФС материалов, отражающей процесс преобразования структуры ИИИС, и созданных продукционных правил. Соответствующие модели различных структур ИИИС ТФС материалов отображают вершины графа $E_{B i}, i=1, \ldots, 3$. На рис. 2 обозначены модели различных структур ИИИС: типовой $-E_{B 1}$; для определения ТФС материалов с низкой теплопроводностью $-E_{B 2}, \lambda=[(0,03 . . .0,06)$ Вт/мК]; для определения ТФС материалов со средней теплопроводностью $-E_{B 3}, \lambda=[(0,061 \ldots 0,115)$ Вт/мК $]$; для определения ТФС материалов с высокой теплопроводностью $-E_{\text {в4 }}, \lambda=[(0,116 \ldots 0,2)$ Вт $/$ мК $]$.

Модель процедуры изменения конфигурации структуры системы в соответствии с идентификацией измерительной ситуации отображкает оператор преобразования моделей структуры ИИИС $\bar{W}_{i}$, $i=1, \ldots 4$.

Оператор $W$ - приведен в виде кортежа: $\bar{W}=\left\langle V_{0}, V_{e}, Z_{\text {кон }}\right\rangle$, где представлены следующие множества: $V_{0}=\left\{V_{b}^{0}, b=1, \ldots, e_{0}\right\}-$ множество модулей обеспечения при реконфигурировании структуры системы: математического $V_{\text {моbi }}$, программного $V_{\text {поbi, }}$, и метрологического $V_{\text {мет.obi }} ; V_{e}=\left\{V_{b}^{e}, b=1, \ldots, e_{b}\right\}$ - множество структурных модулей ИИИС, в которые входят различные структурные компоненты системы: базы данных и знаний, микроконтроллеры, интеллектуальные измерительные датчики и др.; $Z_{\text {кон }}$ - алгоритм реконфигурирования структуры ИИИС ТФС материалов с использованием созданных продукционных правил: $R_{\text {пр }}=\left\{R_{\text {пр1 }}, \ldots, R_{\text {пр4 }}\right\}$.

Реконфигурирование структуры ИИИС выполняется с помощью оператора $W$ - и созданных продукционных правил $R_{\text {пр1 }}, \ldots, R_{\text {пр4 }}$. Модель $E_{B 1}$ и правило $R_{\text {пр1 }}$ формируют типовую структуру системы; применение правила $R_{\text {пр2 }}$ позволяет определить структуру системы в соответствии с моделью $E_{B 2}$; модель $E_{B 3}$ используется для создания структуры

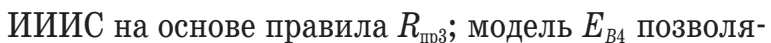
ет формировать структуру системы с использованием правила $R_{\text {пр1 }}$. Согласно алгоритму реконфигурирования ИИИС ТФС материалов и созданным продукционным правилам, преобразование моделей структуры $E_{B i}, i=1, \ldots, 4$ с использованием операторов выполняется следующим образом:

$$
\begin{aligned}
& \bar{W}_{1} \rightarrow Z_{\text {кон } 1} \rightarrow\left\{R_{\text {пр1 }}\right\} ; \bar{W}_{2} \rightarrow Z_{\text {кон } 2} \rightarrow\left\{R_{\text {пр } 2}\right\} ; \\
& \bar{W}_{3} \rightarrow Z_{\text {кон } 3} \rightarrow\left\{R_{\text {пр } 3}\right\} ; \bar{W}_{4} \rightarrow Z_{\text {кон } 4} \rightarrow\left\{R_{\text {пр } 4}\right\} .
\end{aligned}
$$

Методика идентификации излерительной ситуаиии.

Определение измерительной ситуации проводится путем предварительной идентификации свойств исследуемого материала на основании байесовского подхода к обработке выходных сигналов структурных компонентов измерительного канала (ИК) ИИИС - первичных измерительных преобразователей - измерительных датчиков (ИД), усилителя (У) и аналого-цифрового преобразователя (АЦП) [11-14]. Сущность подхода заключается в назначении $L$ возможных диапазонов значений по (для) каждому $j$-му контролируемому свойству ИМ, вычислении вероятностей нахождения контролируемых свойств в выделенных диапазонах на основе выходных сигналов ИК ИИИС и идентификации по критерию максимума апостериорной вероятности исследуемых свойств.

Модель процесса измерения в ИИИС ТФС материалов имеет вид [15]:

$$
Z_{k}=C_{k} X_{k}+N_{k} \zeta_{k}, \quad k=\overline{1, K},
$$

где $Z_{k}$ - вектор измерений, компоненты которого соответствуют контролируемым свойствам ИМ; $X_{k}$ - вектор контролируемых свойств; $C_{k}$ - матрица наблюдений; $N_{k}$ - заданная матрица, соответствующая точностным характеристикам ИК ИИИС; $\zeta_{k}-$ вектор шумов измерения; $k$ - текущее измерение; $K$ - количество измерений в серии. Алгоритм вычисления вероятностей нахождения кон- 
тролируемых свойств ИМ в выделенных диапазонах, разработанный по аналогии с [16, 17], будет следующий:

$$
\begin{gathered}
\widehat{p}_{k}(l)=\frac{\tilde{p}_{k}(l) \exp \left[-0.5 h_{k}(l)\right]}{\sum_{L} \tilde{p}_{k}(l) \exp \left[-0.5 h_{k}(l)\right]}, \quad l=\overline{1, L} ; \\
\tilde{p}_{k}(l)=\sum_{L} q\left(l_{k} / l_{k-1}\right) \widehat{p}_{k-1}(l), \quad l=\overline{1, L}, k=\overline{1, K} ; \\
h_{k}(l)=\sum_{j=1}^{J} h_{j}^{(l)}\left(x_{j}\right) ; \\
h_{j}^{(l)}\left(x_{j}\right)=\frac{\left(\hat{x}_{j}-m_{j}(l)\right)^{2}}{\widehat{D}_{x j}}, j=\overline{1, J},
\end{gathered}
$$

где $\hat{p}_{k}(l), \tilde{p}_{k}(l)$ - соответственно апостериорная и прогнозируемая вероятности нахождения контролируемого свойства в $l$-м диапазоне при $k$-м измерении; $L$ - количество диапазонов разбиения свойств ИМ; $q\left(l_{k} / l_{k-1}\right)$ - коэффициент прогнозирования числового значения контролируемого свойства ИМ; $\hat{p}_{k-1}(l)-$ апостериорная вероятность нахождения контролируемого свойства в $l$-м диапазоне при предыдущем $k$-1-м измерении. Если есть дополнительная информация для прогнозирования, то $q\left(l_{k} / l_{k-1}\right) \neq 1$, но в общем случае, как правило, такой информации нет, поэтому $q\left(l_{k} / l_{k-1}\right)=1 ; \hat{x}_{j}$, $m_{j}(l), \hat{D}_{x j}$ - соответственно оценка (знак оценки "^» над соответствующим символом) измеренного значения $j$-го свойства, среднее значение $l$-го диапазона, оценка дисперсии выходного сигнала ИК j-го свойства ИМ; $J$ - количество контролируемых свойств исследуемых материалов.

Предварительная идентификация контролируемого свойства выполняется по критерию максимума апостериорной вероятности по результатам серии из $K$ измерений

$$
\hat{l}_{K}=\arg \max \widehat{p}_{K}(l) .
$$

Алгоритм (2)-(6) совместно с известными алгоритмами калмановской фильтрации для оценивания выходных измерительных сигналов ИК ИИИС [18-20] может использоваться в качестве базового при предварительной идентификации контролируемых свойств известных ИМ. В случае отсутствия каких-либо сведений об ИМ для повышения достоверности «первичных» измерений матрицу наблюдений в модели (1) целесообразно формировать по методологии управляемого эксперимента [21]. В соответствии с этой методологией осуществляется выбор программы (режима) измерений, состава контролируемых свойств, положения (траектории движения) измерительных датчиков. Тогда в модели (1) матрица $C_{k}$ будет зависеть от состава контролируемых свойств и условий их измерения, т. е. приобретает вид $C_{k}\left(\mu_{k}, \gamma_{k}\right)$, где $\mu_{k}$ - матрица состава контролируемых свойств, $\gamma_{k}-$ вектор управления процессом измерения. Множество возможных программ управления процессом измере- ния $\Gamma_{k}$ и количество $J$ контролируемых свойств ИМ - исходные заданные параметры [22].

При выборе программы измерений, связанной с определением моментов времени для измерения, параметры $\gamma_{k}$ и $\mu_{k}$ - скалярные. Множество $\Gamma_{k}$ состоит из двух элементов: $\Gamma_{k}=\{0,1\}$, при этом $\gamma_{k}=1$, если измерение производится; $\gamma_{k}=0$, если измерение отсутствует. Тогда матрица состава контролируемых свойств примет вид $C_{k}\left(\mu_{k}, \gamma_{k}\right)=\gamma_{k} C_{k}$. При выборе состава контролируемых свойств формально $\mu_{k}=\gamma_{k}$, а матрица наблюдений приобретает вид $C_{k}\left(\mu_{k}, \gamma_{k}\right)=\mu_{k} C$.

При выборе положения измерительных датчиков в качестве дополнительной выступает процедура оптимизации их расположения в соответствии с критерием минимума средних потерь minI $\left(\hat{X}_{k}\right)$, обеспечивающего повышение качества оценивания свойств ИМ:

$$
\begin{aligned}
& I\left(\widehat{X}_{k}\right)=M\left[\Psi\left(X_{k}, \widehat{X}_{k}\right)\right]=\int_{-\infty}^{\infty} \Psi\left(X_{k}, \widehat{X}_{k}\right) \widehat{p}_{k}(X) d X ;(7) \\
& \Psi\left(X_{k}, \widehat{X}_{k}\right)=\sum_{j=1}^{J}\left[X_{j k}-\widehat{X}_{j k}\right]^{2},(8)
\end{aligned}
$$

где $M[\bullet]$ - операция математического ожидания; $\hat{p}_{k}(X)$ - апостериорная плотность вероятности вектора контролируемых свойств; $\Psi\left(X_{k}, \hat{X}_{k}\right)$ - квадратичная функция потерь, определяемая в соответствии с (8). Расположение измерительных датчиков будет наилучшим при достижении минимума критерия (7).

С использованием концептуальной модели проектирования ИИИС и модели процесса реконфигурирования структуры системы, приведённых на рис. 1,2 , структурной схемы модулей реконфигурирования ИИИС (рис. 3), создана структурная схема ИИИС ТФС материалов, представленная на рис. 4.

В соответствии с классом исследуемых теплоизоляционных материалов, отличающихся по теплопроводности, разработанная ИИИС на основе базовой структуры путем её реконфигурирования позволяет создать новую структуру. В ИИИС применяются методы искусственного интеллекта (распознавания образов при классификации теплоизоляционных материалов) при выборе метода определения теплофизических свойств теплоизоляционных материалов с адаптацией к классу исследуемых материалов, что позволяет повысить точность результатов измерения. В состав математического обеспечения системы входят ряд созданных математических моделей, применяемых в ИИИС: модель предметной области $E_{\text {по }}$, модель ИИИС $E_{\text {ииис }}$ и принятия решений $E_{\text {П }}$. На этапе разработки математического обеспечения для рассматриваемой предметной области (контроль ТФС материалов) применяются методы искусственного интеллекта для представления знаний (фреймы, нечеткие множества), принятия решений в системе (выбор метода определения ТФС материалов, распознавание образов) и решения задач оптими- 


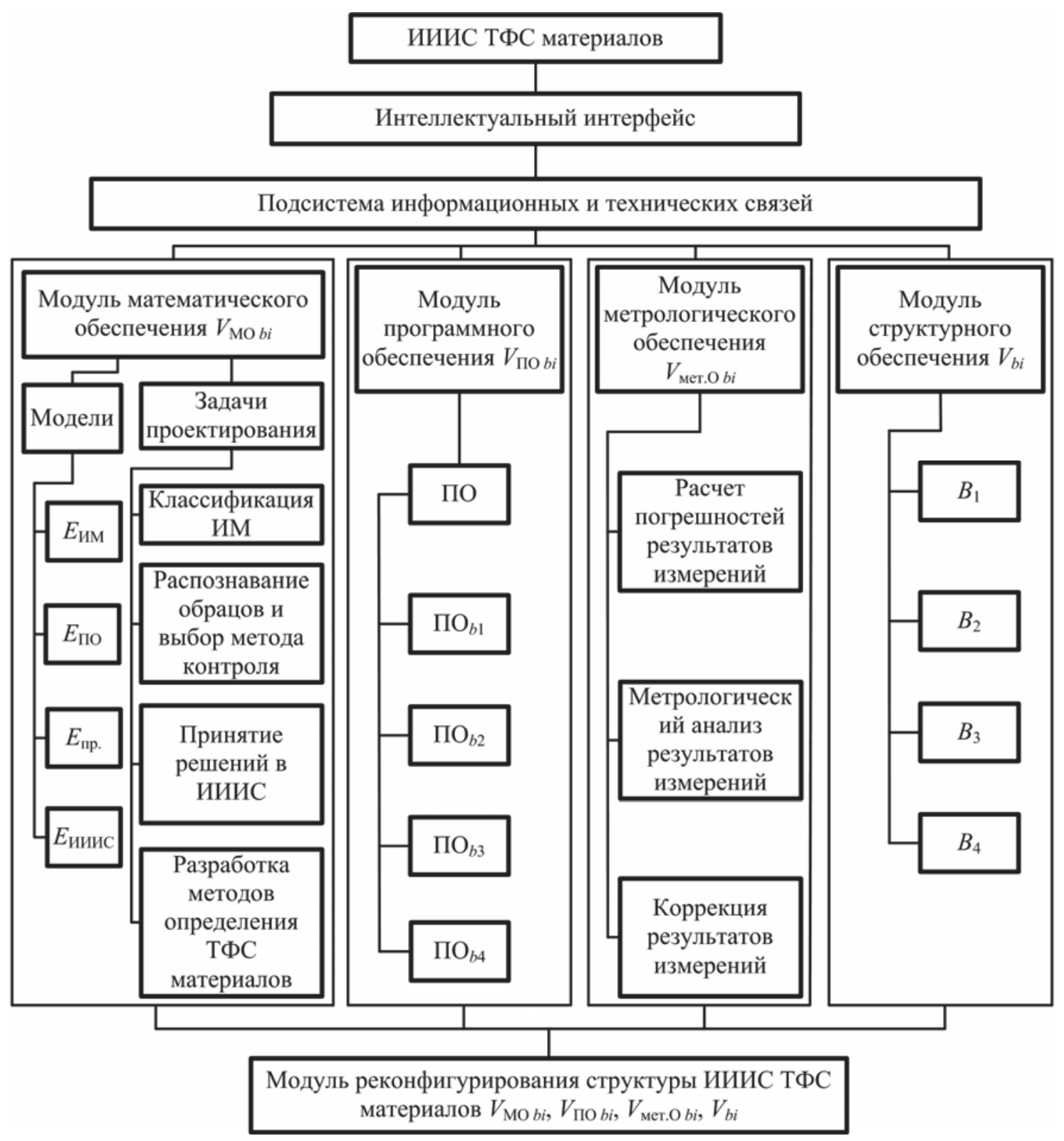

Рис. 3. Структурная схема модулей реконфигурирования ИИИС ТФС материалов

Fig. 3. Block diagram of modules of reconfiguring the IIIS TFS materials

зации режимных параметров теплофизических измерений.

В ИИИС применяется метод определения ТФС материалов с элементами искусственного интеллекта. Метод заключается в снятии тестовой термограммы (температурно-временной зависимости) при помещении измерительного зонда ИИИС на исследуемое теплозащитное покрытие трубопровода или резервуара и осуществлении сформированного теплового воздействия заданной мощности на его поверхность одним из видов нагревателя измерительного зонда. Тестовая термограмма позволяет оценить теплопроводность материала покрытия предварительно. На основе полученных расчетноэкспериментальных температурных зависимостей определяют оптимальные режимные параметры для проведения теплофизических измерений в зависимости от класса теплоизоляционного материала по теплопроводности и последующего расчёта параметров ТФС материалов.

Полученная измерительная информация обрабатывается с использованием разработанного программного обеспечения интеллектуальной информационно-измерительной системы, а также формируется информация в базах знаний и данных, реализуется интеллектуальный интерфейс и алгоритм функционирования ИИИС. В ИИИС осуществляется идентификация измерительной ситуации, по результатам которой выбирается метод определения теплофизических свойств теплозащитных 


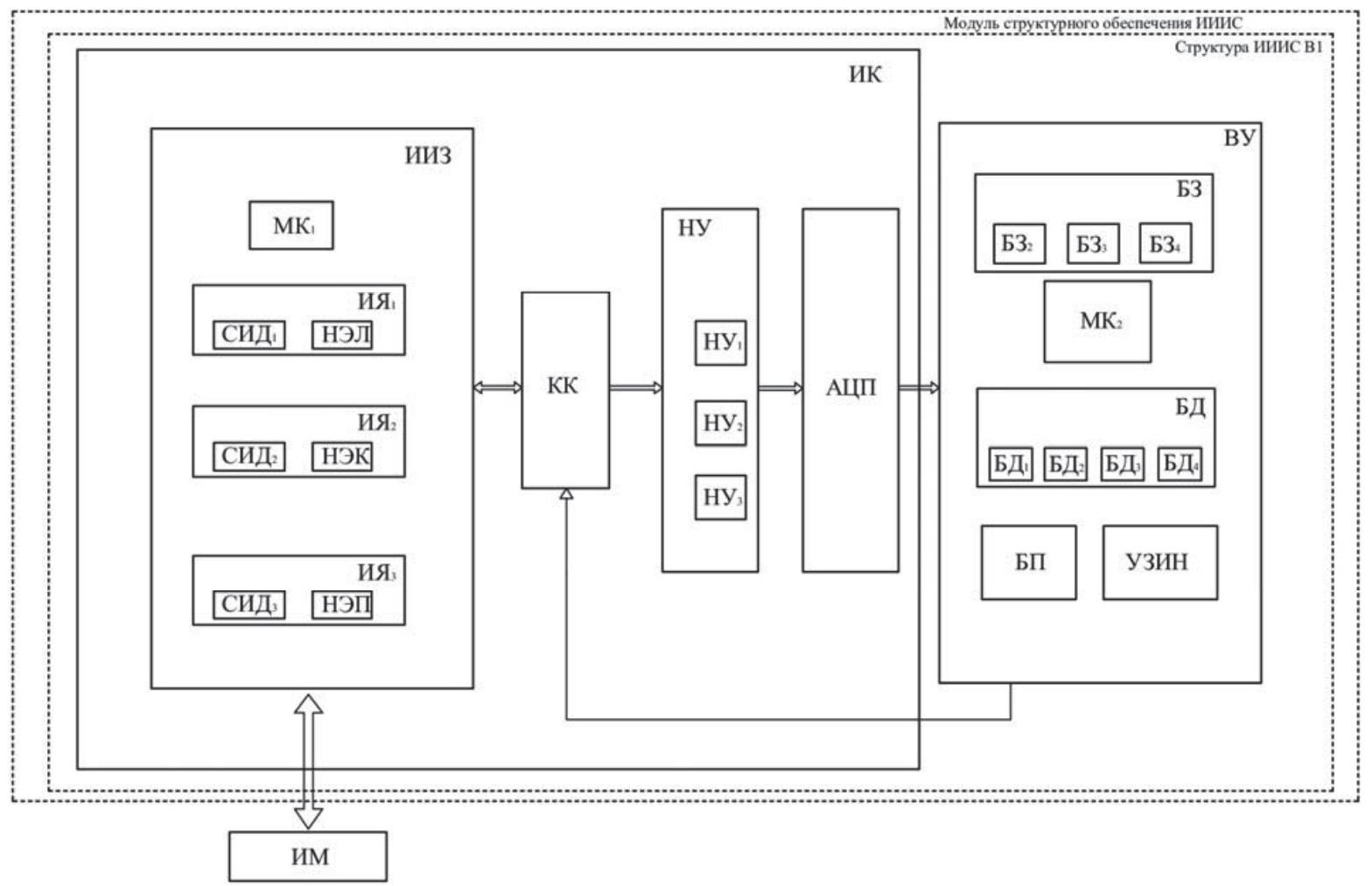

Pис. 4. Структурная схема ИИИС ТФС материалов с реконфигурируемой структурой

Fig. 4. Block diagram of the IIIS TFS of materials with reconfigurable structure

покрытий и оптимальные режимные параметры при проведении теплофизических измерений для повышения метрологического уровня ИИИС. Программа создана с использованием языка программирования $C^{+}$, структура ИИИС ТФС материалов разработана на базе PIC микроконтроллеров фирмы MICROCHIP.

Определение погрешностей и метрологический анализ результатов измерений параметров ТФС материалов, повышение метрологического уровня ИИИС ТФС материалов с использованием автоматической коррекции результатов полученных экспериментальных данных осуществляется с помощью разработанного метрологического обеспечения ИИИС. В базе знаний системы содержатся необходимые данные для того, чтобы провести поверку и испытания созданной системы, а также информация о способах оценки результатов работы ИИИС ТФС материалов - применение критерия Демпстера-Шафера; математических зависимостях для определения погрешностей измерения и их характеристик $[23,24]$.

Для реконфигурирования структуры ИИИС модуль структурного обеспечения $V_{b i}$ содержит: интеллектуальный измерительный зонд (ИИЗ), в который входят измерительные ячейки ИЯ $1,{ }_{1} Я_{2}$, ИЯ $_{3}$ с системой измерительных датчиков СИД СИД, СИД гревательные элементы (линейный НЭЛ, круглый НЭК и плоский НЭП, т. е. соответствующей кон- фигурации в зависимости от класса теплопроводности ИМ для формирования различной мощности теплового воздействия на ИМ при проведении теплофизических измерений), микроконтроллер $\mathrm{MK}_{1}$ в составе измерительного канала (ИК) с коммутатором КК для подключения ИЯ ${ }_{1}, И_{2}, И_{3}$, нормирующими усилителями $\mathrm{HУ}_{1}, \mathrm{HУ}_{2}, \mathrm{HУ}_{3}$ с различными диапазонами усиления и аналого-цифровым преобразователем (АЦП); вычислительное устройство (ВУ), включающее: блок питания (БП), с выхода которого поступают напряжения $U_{1}, U_{2}$, $U_{3}$ при создании структур $B_{2}, \ldots B_{4} ;$ УЗИН - устройство задания импульсов нагрева, использующееся при формировании структур $B_{2}, \ldots B_{4}$; базы знаний Б3 ${ }_{2}-$ Б3 $_{4}$, базы данных БД - БД рых используется при создании структур $B_{1}, \ldots B_{4}$.

Структура ИИИС должна быть многофункциональной и универсальной, реализующей в автоматизированном режиме алгоритм функционирования системы и принятия решений в условиях неопределённости, применение методов искусственного интеллекта при формировании базы знаний ИИИС, распознавания образов (класса исследуемых материалов). Данные свойства системы реализуются на основе использования модулей $B_{2}, B_{3}$, $B_{4}$, при этом применяются интеллектуальные процедуры реконфигурирования модульной типовой структуры $B_{1}$ ИИИС согласно правилам $R_{\text {пр1 }}, \ldots, R_{\text {пр }}$. Структура $B_{1}$ включает измерительный канал, в состав которого входит интеллектуальный измери- 
тельный зонд, включающий измерительные ячейки $И Я_{1}, И_{2}, И_{3}$ при формировании, соответственно, структур $B_{2}, B_{3}, B_{4}$; вычислительное устройство с микроконтроллером $\mathrm{MK}_{2}$, а также базы данных и знаний, в которые записана следующая информация: математические модели используемых теплоизоляционных материалов, алгоритмы реализации методов измерения параметров теплофизических свойств теплозащитных покрытий трубопроводов, измерительные процедуры, сведения о предметной области исследования, измерительных ситуациях, аппроксимирующие математические зависимости результатов измерения от воздействующих дестабилизирующих факторов.

таблица. Относительная погрешность определения коэффи ииента теплопроводности теплозащитных покрытий трубопровода (резервуара)

Table. Relative error in determining heat conductivity coefficient of heat-shielding coverings of the pipeline (tank)

\begin{tabular}{|c|c|c|c|}
\hline \multirow[t]{2}{*}{$\begin{array}{c}\text { Теплозащитные } \\
\text { покрытия трубопровода } \\
\text { Heat-shielding coverings } \\
\text { of pipeline }\end{array}$} & $\begin{array}{c}\text { Справочные } \\
\text { данные } \\
\text { Help data }\end{array}$ & \begin{tabular}{|} 
Измеренные \\
данные \\
Measured \\
data
\end{tabular} & \begin{tabular}{|c} 
Относительная \\
погрешность \\
измерения \\
Relative measu- \\
rement error
\end{tabular} \\
\hline & $\begin{array}{c}\lambda, \mathrm{BT} /(\mathrm{mK}) \\
\mathrm{W} / \mathrm{mK})\end{array}$ & $\begin{array}{c}\lambda, \mathrm{B} \text { T/(mK) } \\
\mathrm{W} /(\mathrm{mK})\end{array}$ & $\delta_{\lambda}, \%$ \\
\hline $\begin{array}{l}\text { Пенополиуретан } \\
\text { Polyurethane foam }\end{array}$ & 0,029 & 0,030 & 3,45 \\
\hline $\begin{array}{l}\text { Минеральная вата } \\
\text { Mineral wool }\end{array}$ & 0,040 & 0,0415 & 3,75 \\
\hline $\begin{array}{l}\text { Маты из стекловолокна } \\
\text { Mats from fiber glasses }\end{array}$ & 0,050 & 0,052 & 4,00 \\
\hline $\begin{array}{l}\text { Жесткая минераловат- } \\
\text { ная плита на битумном } \\
\text { связующем } \\
\text { Rigid mineral-cotton pla- } \\
\text { te on bituminous binding }\end{array}$ & 0,070 & 0,073 & 4,29 \\
\hline $\begin{array}{l}\text { Рубероид } \\
\text { Roofing material }\end{array}$ & 0,170 & 0,178 & 4,70 \\
\hline $\begin{array}{l}\text { Пенобетон } \\
\text { Foam concrete }\end{array}$ & 0,210 & 0,220 & 4,76 \\
\hline
\end{tabular}

Синтез структуры ИИИС выполняется в зависимости от результатов идентификации измери-

\section{СПИСОК ЛИТЕРАТУРЫ}

1. Р 536-84. Рекомендации по проектированию теплоизоляционных конструкций магистральных трубопроводов. - М.: Всесоюзный научно-исследовательский институт по строительству и эксплуатации трубопроводов, объектов ТЭК - инжиниринговая нефтегазовая компания, 1985. - 54 с.

2. Selivanova Z.M., Khoan T.A. A Systematic Method of Improving the Accuracy of an Information and Measuring System for Determining the Thermophysical Properties of Materials Under the Effect of Destabilizing Factors // Measurement Techniques. 2017. - V. 60. - Iss. 5, August. - P. 473-480.

3. Стасенко K.С., Селиванова 3.М. Интеллектуальная информационно-измерительная система мониторинга режимных параметров технологического процесса производства минеральных ват // Вестник Тамбовского государственного технического университета. - 2013. - Т. 19. - № 1. - С. 52-60.

4. Belyaev V.P., Mischenko S.V., Belyaev P.S. Determination of the Diffusion Coefficient in Nondestructive Testing of Thin Articles тельной ситуации с использованием модели процесса реконфигурирования структуры ИИИС ТФС материалов (рис. 2).

Проведены экспериментальные исследования созданной ИИИС ТФС материалов, результаты которых приведены в таблице.

Как показывают данные экспериментальных исследований интеллектуальной информационноизмерительной системы ТФС теплоизоляционных материалов, относительная погрешность результатов измерения коэффициента теплопроводности теплозащитных покрытий с применением разработанной ИИИС составляет не более $5 \%$.

\section{Заключение}

Предложена концептуальная модель проектирования интеллектуальной информационно-измерительной системы теплофизических свойств теплозащитных покрытий магистральных трубопроводов и резервуаров для транспортировки и хранения нефти и газа, методика идентификации измерительной ситуации и модель реконфигурирования структуры ИИИС в результате реализации интеллектуальной процедуры преобразования модулей структурного, математического, метрологического и программного обеспечения для повышения точности определяемого параметра ТФС исследуемых материалов в ИИИС коэффициента теплопроводности.

Полученные результаты рекомендуется использовать при создании ИИИС определения ТФС теплоизоляционных материалов в диапазоне теплопроводности от 0,02 до 0,2 Вт/мК.

Работа выполнена при финансовой поддержке Министерства науки и высшего образования РФ (государственное задание, проект "Интеллектуальная информационно-измерительная и управляюшая система оперативного контроля теплофизических характеристик теплоизоляиионных материалов, применяемых в условиях Арктики. Разработка теоретических основ, методологии построения интеллектуальных информационно-измерительных и управляющих систем»).

of Anisotropic Porous Materials // Measurement Techniques. 2017. - V. 60. - Iss. 4, 1 July. - P. 392-398.

5. Maglic D.K. Stationary methods for the measurement of thermophysical properties // High Pressures. - 1979. - V. 11. № 11. - P. 1-8.

6. The development of the data transfer protocol in the intelligent control systems of the energy carrier parameters / I.S. Karavaev, V.I. Selivantsev, Y.I. Shtern, M.Y. Shtern // Proceedings of the IEEE Conference of Russian Young Researchers in Electrical and Electronic Engineering, ElConRus. - Saint Petersburg, January 29-31, 2018. - P. 1305-1308.

7. Development of the method of software temperature compensation for wireless temperature measuring electronic instruments / Y.I. Shtern, I.S. Karavaev, M.Y. Shtern, M.S. Rogachev // International Journal of Control Theory and Applications. - 2016. № 9. - P. 139-146.

8. Советов Б.Я., Яковлев С.А. Моделирование систем. - М.: Высш. шк., 2005. - 343 с. 
9. Ахремчик 0.Л. Информационная база для автоматизированного проектирования схем систем управления технологическими объектами // Информационные технологии. - 2009. № 8. - C. 17-21.

10. Уилсон Р. Введение в теорию графов. - М.: Мир, 1977. - 208 с.

11. Овчаренко В.Н. Планирование идентифицирующих входных сигналов в линейных динамических системах // Автоматика и телемеханика. - 2001. - № 2. - С. 75-87.

12. Сейдж Э.П., Мелса Дж.Л. Идентификация систем управления. - М.: Наука, 1974. - 248 с.

13. Lindquist A., Pieci G. On «Subspace Methods» Identification and Stochastic Model reduction // Proc. of SYSID'94. - Copenhagen, Denmark, 4-6 July, 1994. - V. 2. - P. 397-404.

14. Ljung L. Issues in System Identification // IEEE Control Systems. - 1991. - V. 11. - № 1. - P. 25-29.

15. Аксенова Т.В., Павлов В.И., Аксенов В.В. Алгоритмическое обеспечение информационно-измерительной системы мониторинга технического состояния трубопроводов // Химическое и нефтегазовое машиностроение. - 2015. - № 8. - С. 39-42.

16. Павлов В.И., Аксенова Т.В., Аксенов В.В. Эффективность информационной поддержки оператора при управлении объектом энергетики // Известия Томского политехнического университета. - 2015. - Т. 326. - № 3. - С. 70-76.

17. Распознавание состояния информационно-измерительной системы при неточных измерениях вектора ее фазовых координат / Г.Г. Себряков, С.М. Мужичек, А.А. Скрынников, В.И. Павлов, О.В. Ермолин // Вестник компьютерных и информационных технологий. - 2016. - № 11. - С. 15-19.

18. Zhang $\mathrm{Q}$. Adaptive Kalman filter for actuator fault diagnosis // Automatica. - 2018. - V. 93, 1 July. - P. 333-342.
19. Sensitivity Analysis of Extended and Unscented Kalman Filters for Attitude Estimation / M. Rhudy, Y. Gu, J. Gross, S. Gururajan, M. Napolitano // AIAA Journal of Aerospace Information Systems. - 2013. - V. 10. - № 3. - P. 131-143.

20. Wan E., Van der Merwe R. The Unscented Kalman Filter. Ch. 7 // Kalman Filtering and Neural Networks. - New York, Wiley, 2002. - P. 221-282.

21. Малышев В.В., Красильщиков М.Н., Карлов В.И. Оптимизация наблюдения и управления летательных аппаратов. - М.: Машиностроение, 1989. - 312 с.

22. Airborne data measurement system errors reduction through state estimation and control optimization / G.G. Sebryakov, S.M. Muzhichek, V.I. Pavlov, O.V. Ermolin, A.A. Skrinnikov // 2018 Workshop on Materials and Engineering in Aeronautics (MEA2018). - Moscow, Moscow Technological Institute. Publ., 2018. - P. 1-5.

23. Optimal design for the room temperature control and household heat metering system. Advanced Materials Research / M. Fu, Y.J. Zhang, J.D. Ye, J.Y. Jiang, F. Zhang // $2^{\text {nd }}$ International Conference on Energy and Environmental Protection, ICEEP 2013. - Guilin, China, 19-21 April 2013. - V. 724-725. P. 969-975.

24. Design and implementation of wireless monitoring network for temperature-humidity measurement / W. Guan, C. Wang, Y. Cai, H. Zhang // Journal of Ambient Intelligence and Humanized Computing. - February 2016. - V № 7 (1). - P. 131-138.

Поступила 14.02.2019 2.

\section{Информация об авторах}

Селиванова 3.М., доктор технических наук, профессор кафедры конструирования радиоэлектронных и микропроцессорных систем Тамбовского государственного технического университета.

Павлов В.И., доктор технических наук, профессор кафедры конструирования радиоэлектронных и микропроцессорных систем Тамбовского государственного технического университета. 
UDC 66.067.55

\title{
INTELLIGENT INFORMATION-MEASURING SYSTEM OF THERMOPHYSICAL PROPERTIES OF THERMAL INSULATION COATINGS OF TRUNK PIPELINES
}

\author{
Zoya M. Selivanova', \\ selivanova@mail.jesby.tstu.ru \\ Vladimir I. Pavlov', \\ vpav|@mail.ru \\ 1 Tambov State Technical University, \\ 106, Sovetskaya street, Tambov, 392000, Russia.
}

Relevance of the research. In the main pipelines for transportation as well as in tanks for oil and gas storage the heat-shielding coverings from various materials are applied. The heat-shielding of these objects is inevitably intensively affected by various climatic factors, and in certain cases by mechanical influence that leads to degradation of its properties. Development of intellectual information and measuring system with reconfigurable structure for operating control of heat-insulating materials condition, which allows expanding the functionality of the existing similar systems and providing the required accuracy of measurements, is important and relevant.

The aim of the research is to increase the accuracy in determining the coefficient of heat conductivity of various heat-shielding coverings of the main pipelines and tanks by their production and operation for prevention of deterioration in properties of thermal insulation under the influence of the external influencing factors.

Objects: heat-shielding coverings of the main pipelines and tanks used in oil and gas transporting and storage.

Methods. The methodological basis at solution of objectives of design of intellectual information and measuring system of heatphysical properties of materials consists in: theory of measuring systems, artificial intelligence methods, Bayesian approach.

Results. The authors have developed the conceptual model of designing the intellectual information and measuring system of heatphysical properties of heat-insulating coverings of the main pipelines for oil and gas transportation and tanks at storage. Within the conceptual model the authors developed the technique for defining a measuring situation in heatphysical measurements on the basis of preliminary identification of properties of the studied material using the Bayesian approach to processing the output signals of primary measuring converters and structural components of the measuring channel of system. The authors designed the model of reconfiguring and the relevant structure of intellectual information and measuring system of heatphysical properties of heat-insulating coverings to increase the accuracy of determination of coefficient of heat conductivity of heat-insulating materials. The relative error of measurement of coefficient of heat conductivity when using the offered system makes no more than $5 \%$.

Keywords:

Conceptual model of design, Bayesian approach, measurement model, main pipeline, tank.

This work was financially supported by the Ministry of Science and Higher Education of the Russian Federation (state task, the project «Intelligent information-measuring and control system for the operational control of the thermophysical characteristics of heat-insulating materials used in the Arctic. Development of theoretical foundations, methodology for constructing intelligent information-measuring and control systems»).

\section{REFERENCES}

1. R 536-84. Rekomendatsii po proektirovaniyu teploizolyatsionnykh konstruktsiy magistralnykh truboprovodov [P 536-84. Recommendations for the design of thermal insulation structures of main pipelines]. Moscow, All-Union Scientific Research Institute for the construction and operation of pipelines, fuel and energy facilities - engineering oil and gas company Publ., 1985. $54 \mathrm{p}$.

2. Selivanova Z.M., Khoan T.A. A Systematic Method of Improving the Accuracy of an Information and Measuring System for Determining the Thermophysical Properties of Materials under the Effect of Destabilizing Factors. Measurement Techniques, 2017, vol. 60 , no. 5, pp. 437-480.

3. Stasenko K.S., Selivanova Z.M. Intellektualnaya informatsionnoizmeritelnaya sistema monitoringa rezhimnykh parametrov tekhnologicheskogo protsessa proizvodstva mineralnykh vat [Intelligent information-measuring system for monitoring the regime parameters of the technological process for the production of mineral wool]. Bulletin of Tambov State Technical University, 2013, vol. 19, no. 1, pp. 52-60.

4. Belyaev V.P., Mischenko S.V., Belyaev P.S. Determination of the Diffusion Coefficient in Nondestructive Testing of Thin Articles of Anisotropic Porous Materials. Measurement Techniques, 2017, vol. 60, no. 4, pp. 392-398. In Rus.
5. Maglic D.K. Stationary methods for the measurement of thermophysical properties. High Pressures, 1979, vol. 11, no. 11, pp. 1-8.

6. Karavaev I.S., Selivantsev V.I., Shtern Y.I., Shtern M.Y. The development of the data transfer protocol in the intelligent control systems of the energy carrier parameters. Proc. of the IEEE Conference of Russian Young Researchers in Electrical and Electronic Engineering. St- Petersburg, January, 29-31, 2018. pp. 1305-1308.

7. Shtern Y.I., Karavaev I.S, Shtern M.Y., Rogachev M.S. Development of the method of software temperature compensation for wireless temperature measuring electronic instruments. International Journal of Control Theory and Applications, 2016. vol 9. pp. 139-146.

8. Sovetov B.Ya., Yakovlev S.A. Modelirovanie sistem [Modeling of Systems]. Moscow, Vysshaya shkola Publ., 2005. 343 p.

9. Akhremchik 0.L. Informatsionnaya baza dlya avtomatizirovannogo proyektirovaniya skhem sistem upravleniya tekhnologicheskimi obyektami [Information base for computer-aided design of schemes for control systems of technological objects]. Moscow, Information technologies Publ., 2009. No. 8, pp. 17-21.

10. Uilson R. Vvedenie $v$ teoriyu grafov [Introduction to graph theory]. Moscow, Mir Publ., 1977. 208 p. 
11. Ovcharenko V.N. Planirovanie identifitsiruyushchikh vkhodnykh signalov $v$ lineynykh dinamicheskikh sistemakh [Planning of identifying input signals in linear dynamic systems]. Moscow, Avtomatika i telemekhanika Publ., 2001. No. 2, pp. 75-87.

12. Seydzh E. P., Melsa Dzh. L. Identifikatsiya sistem upravleniya [Identification of control systems]. Moscow, Nauka Publ., 1974. $248 \mathrm{p}$.

13. Lindquist A., Pieci G. On «Subspace Methods» Identification and Stochastic Model reduction. Proc. of SYSID '94. Copenhagen, Denmark, 4-6 July, 1994. Vol. 2, pp. 397-404.

14. Ljung L. Issues in System Identification. IEEE Control Systems, 1991, vol. 11, no. 1, pp. 25-29.

15. Aksenova T.V., Pavlov V.I., Aksenov V.V. An Algorithm-Based Program of an Information and Measurement System for Monitoring the Technical State of Pipelines. Chemical and Petroleum Engineering, 2015, vol. 51, Iss. 7-8, pp. 565-568. In Rus.

16. Pavlov V.I., Aksenova T.V., Aksenov V.V. Efficiency of information support of operator when controlling power engineering facilities. Bulletin of the Tomsk Polytechnic University, 2015, vol. 326, no. 3, pp. 70-76. In Rus.

17. Sebryakov G.G., Muzhichek S.M., Skrinnikov A.A., Pavlov V.I., Ermolin 0.V. Recognition of information-measuring systems states during inaccurate measurement of its phase coordinates vector. Herald of computer and information technologies. Scientific, technical and production monthly journal, 2016, no. 11, pp. 15-19. In Rus.

18. Zhang Q. Adaptive Kalman filter for actuator fault diagnosis. $A u$ tomatica, July 2018, vol. 93, pp. 333-342.
19. Rhudy M., Gu Y., Gross J., Gururajan S., Napolitano M. Sensitivity Analysis of Extended and Unscented Kalman Filters for Attitude Estimation. AIAA Journal of Aerospace Information Systems, March 2013, vol. 10, no. 3, pp. 131-143.

20. Wan E., Van der Merwe R. The Unscented Kalman Filter. Ch. 7. Kalman Filtering and Neural Networks. New York, Wiley, 2002. pp. 221-282.

21. Malyshev V.V., Krasilshchikov M.N., Karlov V.I. Optimizatsiya nablyudeniya $i$ upravleniya letatelnykh apparatov [0ptimization of observation and control of aircrafts]. Moscow, Mashinostroenie Publ., 1989. 312 p.

22. Sebryakov G.G., Muzhichek S.M., Pavlov V.I., Ermolin O.V., Skrinnikov A.A. Airborne data measurement system errors reduction through state estimation and control optimization. Workshop on Materials and Engineering in Aeronautics (MEA2018). Moscow, Moscow Technological Institute Publ., 2018. pp. 1-5.

23. Fu M., Zhang Y.J., Ye J.D., Jiang J.Y., Zhang F. Optimal design for the room temperature control and household heat metering system. Advanced Materials Research. $2^{\text {nd }}$ International Conference on Energy and Environmental Protection, ICEEP 2013. Guilin, China, 19-21 April 2013. Vol. 724-725, pp. 969-975.

24. Guan W., Wang C., Cai Y., Zhang H. Design and implementation of wireless monitoring network for temperature-humidity measurement. Journal of Ambient Intelligence and Humanized Computing, February 2016, vol. 7, no. 1, pp. 131-138.

Received: 14 February 2019.

\section{Information about the authors}

Zoya M. Selivanova, Dr. Sc., professor, Tambov State Technical University.

Vladimir I. Pavlov, Dr. Sc., professor, Tambov State Technical University. 